\title{
Postoperative Outcome and Predictors of Mortality in Octogenarians Patients with Gastric Cancer
}

\author{
Marialuisa Lugaresi ${ }^{1}$, Alessandro Duchi ${ }^{1}$, Daniele Paglione ${ }^{1}$, Francesca Argento ${ }^{1}$, Francesca Martini ${ }^{1}$, \\ Matteo Novello ${ }^{1}$, Salomone Di Saverio ${ }^{2}$ and Bruno Nardo ${ }^{1 *}$ \\ ${ }^{1}$ S Orsola-Malpighi Hospital, Department of Experimental, Diagnostics and Specialty Medicine, Italy \\ ${ }^{2}$ Cambridge Colorectal Unit, Addenbrooke's Hospi-tal, Cambridge Biomedical Campus, Hills Road, Cambridge, England
}

*Corresponding author: Bruno Nardo, Department of Experimental, Diagnostics and Specialty Medicine- Via G. Massarenti 9, 40138 Bologna, Italy

\section{ARTICLE INFO}

Received: 蔧 January 06, 2020

Published: 幽 January 21, 2020

Citation: Marialuisa Lugaresi, Alessandro Duchi, Daniele Paglione, Francesca Argento, Francesca Martini, Matteo Novello, Salomone Di Saverio, Bruno Nardo. Postoperative Outcome and Predictors of Mortality in Octogenarians Patients with Gastric Cancer. Biomed J Sci \& Tech Res 24(5)-2020. BJSTR. MS.ID.004106.

Keywords: Gastric Cancer; Oncology; General Surgery; Gastric Surgery; Geriatric Surgery

Abbreviations: OS: Overall survival; TNM: Tumor Node Metastases; IQR: Inter-Quartile Range

\section{ABSTRACT}

Background: Gastric cancer is most frequent after the fifth decade of life. Surgical risk is higher in aged population be-cause of general health condition may affect the postoperative result. Aim of the study was to identify risk factors for post-operative mortality in octogenarian patients who underwent surgery for gastric cancer.

Methods: 236 patients (181: 80-85 years old and 55: >85 years old) underwent surgery for gastric cancer at the Sant'Orsola-Malpighi University Hospital in Bologna between 2012 and 2017. The variables of the two groups of pa-tients were compared.

Results: Post-operative mortality was 5.5\% among 80-85 years old and 9\% for the $>85$ years old. The two groups sig-nificantly differed in: age $(p<0.0001)$, type of hospitalization $(p=0.005)$, site of primary tumor (pylorus $p=0.030$; body $p=0.001$ ), presence of cardiac comorbidities $(p=0.043)$, ASA score $(p=0.021)$ and type of surgical presentation (elective vs urgent surgery, $p=0.001$ ). In the multivariate analysis, urgent surgery $(p=0.002)$ and ASA score $>$ III $(p=0.021)$ were associated to post-operative mortality; ASA score $(p=0.041)$ and stage $(p=0.012)$ significantly influ-enced survival.

Conclusion: ASA score>III and urgent surgery, but not age $\geq 85$ years, were associated with postoperative mortality. In the very elderly, alias oldest old patients, preoperative nutritional status and pre-existing comorbidities, rather than age itself, should be considered as selection criteria for surgery. ASA risk assessment may be beneficial for stratification of patients and for ultimately optimizing outcomes.

\section{Introduction}

Gastric cancer is the fifth most common malignancy in men and the second in women, with 952,000 new cases diag-nosed per year worldwide [1]. In Italy gastric cancer represent the fifth most common malignancy in the men and the sixth in women, with 14.220 new cases diagnosed in the 2013 year [2]. The treatment of choice for gastric cancer is surgical resection and is both curative and preventive of gastric cancer re-lated complications. Gastric cancer most often occurs in the elderly: at the time of diagnosis $60 \%$ of patients are over the age of 65 [3]. Foreseeing by the Global Health and Aging report, issued by the WHO, predicts that between 2010 and 2050, the world's population of over-85 years old, alias "the oldest old", will increase by $351 \%$ [4]. In Italy has been estimated that the people over 80 years old will be grown up from $3.2 \%$ of 2015 to $7.8 \%$ of 2050 , with and life expectance in that period of 86,6 years for the men and 91.5 years for the women (ISTAT data) [5]. In this context, it is possible to hypothesize that the amount of oldest old patients needing gastric cancer surgery in the next future will be remarkably larger than today. This study aimed to identify risk factors for postoperative mortality in 80-85 years old and $>85$ years old patients who underwent surgical treatment for gastric cancer. For the oldest old patients undergoing gastric cancer surgery, identifica-tion of these factors might lead to a more precise risk stratification and optimization of surgical outcomes. 


\section{Methods}

\section{Inclusion Criteria}

A total of 236 patients aged 80 years or older with histologically confirmed primary gastric cancer underwent surgery in Sant'Orsola-Malpighi Hospital in Bologna between 2012 and 2017. The sample included elective and urgent surgery (both resections and palliative surgery). Clinico-pathological data for these patients were obtained from hospital rec-ords. Comprehensive informed consent was obtained from all patients when they admitted our hospital prior to sur-gery.

\section{Definition and Classification of Variables}

We divided the patients into two categories according to the age: from 80 to 85 years or $>85$ years. The site of primary tumor was categorized as "cardias," "pylorus," "body," "bottom", "not specified or "other". The staging of the tumor was assigned according to the TNM (Tumor Node Metastases) classification of the American Joint Committee on Cancer/Union for International Cancer Control [6,7]. We recorded the ASA score referring to the classification of the American Society of Anesthesiologists [8]. The type of surgical presentation was categorized as "elective" or "urgent." Surgical operation was classified as "partial gastrectomy with jejunal anastomosis," "total gastrectomy," "gastroenter-ostomy without gastrectomy" or "other" (palliative surgery). Surgical approach was categorized as "Roux," "Billroth II" or "other." Postoperative complications were defined as events that occurred during hospitalization following the operation and were evaluated according to Clavien-Dindo classification. Clavien score was referred to Clavien-Dindo classification of surgical complications [9]. Length of hospitalization was defined as the number of days from the date of surgical intervention to discharge. Post-operative mortality was defined that occurred within 30 days from surgery. In-hospital mortality was defined that occurred during hospitalization up to 90 days excluded post-operative mortality. Overall survival (OS) was defined as the time from the date of surgery to patient death (including surgery-associated death or hospital death), or the date of last available information concerning vital status.

Tumor location, clinical or pathological stage, degree of lymph node dissection (D0, <D2 or >D2), and curability were assessed according to the Japanese Classification of Gastric Carcinoma, $13^{\text {th }}$, and then $14^{\text {th }}$ editions $[6,10,11]$. Surgical mortality, morbidity, and hospital mortality were compared between two groups. Recurrences were confirmed by computed tomography, tumor markers, and endoscopic examinations.

\section{Statistical Analysis}

The sample was divided into two cohorts: patients from 80 to 85 and $>85$. Data are represented as median and inter-quartile range (IQR) for continuous variables and as $\mathrm{n}(\%)$ for categorical variables. The $\chi 2$ test or Fisher's test and the Mann-Whitney test were used to analyse categorical and continuous variables. Multivariable logistic regression analysis was performed to identify the predictors of mortality on the combined cohorts. Only variables found to be statistically significant in the univariate analysis were entered into the multivariate model. Survival analysis was performed using the Kaplan-Meier method and the log-rank test. A multivariable (forward stepwise conditional method) Cox regression analysis was performed to estimate the effects of clinical and pathological parameters on survival. In the stepwise procedure, significance levels of .05 for entering and .10 for removing the respective explanatory variables were used to determine the independent risk factors. P-values <.05 were considered significant. Data were analysed using SPSS (version 15.0) (SPSS Inc., Chicago, IL, USA).

\section{Results}

\section{Characteristics of the Patients}

The following tables show a comparison of the variables in detail (Tables 1-4). Among the 80-85 years old ( $n=181)$ and $>85$ years old $(\mathrm{n}=55)(\mathrm{p}<0.0001)$, the type of hospitalization $(\mathrm{p}=$ 0.005 ), the site of primary tumor (pylorus $p=0.030$; body $p=$ 0.001), the presence of cardiac comorbidities ( $p=0.043$ ), the ASA score $(p=0.021)$ and the type of surgical presentation $(p=0.001)$ were proved to be significantly different. The cohort of $>85$ years old had a worse ASA score, a higher percentage of elective surgical interventions (87.3\%) and a higher rate of postoperative mortality (9\%). The 80-85 years old group had a larger number of urgent surgeries (65.2\%). Statistically significant differences between the two groups with regards to the frequency of cardiac comorbili-ties $31.8 \%$ (80-85 years old group) versus 39\% (>85 years old group) and with regards to the site of primary tumor (py-lorus: $80-85$ years old group $44.3 \%$ versus $>85$ years old group $61.9 \%$; body: 80-85 years old group $35.3 \%$ versus $12.7 \%>85$ years old group) were detected. Among 80-85 years old and >85 years old, there were no significant differ-ences $(p>0.05)$ regarding sex, anemia, hypoalbuminemia, depth of cancer invasion, histological type, lymph node me-tastasis, lymph nodes dissection, curative resection, combined resection, tumor stage, type of operation, surgical approach and rates of postoperative complications, Clavien score and post-intervention hospital stay days. 
Table 1: Demographics and clinical and characteristics of octogenarian's patients with gastric cancer.

\begin{tabular}{|c|c|c|c|}
\hline & $\begin{array}{l}\text { Patients } 80-85 \text { years } \\
\qquad(\text { No. }=181)\end{array}$ & $\begin{array}{c}\text { Patients > } 85 \\
\text { years } \\
(\text { No. }=55)\end{array}$ & $P$ value \\
\hline & $82(81-83)$ & $88(86-90)$ & $<0.0001$ \\
\hline & $\begin{array}{l}90(49.7 \%) \\
91(50.3 \%) \\
\end{array}$ & $\begin{array}{l}22(40 \%) \\
33(60 \%) \\
\end{array}$ & 0.281 \\
\hline Anemia: hemoglobin $<10 \mathrm{~g} / \mathrm{dL}$ & $50(27.6 \%)$ & $18(32.7 \%)$ & 0.498 \\
\hline Hypoalbuminemia $<3.5 \mathrm{~g} / \mathrm{dL}$ & $85(46.9 \%)$ & $27(52.9 \%)$ & 0.878 \\
\hline $\begin{array}{c}\text { Hospitalization } \\
\text { Elective } \\
\text { Urgent }\end{array}$ & $\begin{array}{c}118(65.2 \%) \\
63(34.8)\end{array}$ & $\begin{array}{l}24(43.6 \%) \\
21(56.4 \%)\end{array}$ & 0.005 \\
\hline $\begin{array}{c}\text { Comorbidities } \\
\text { Cardiac } \\
\text { Hypertension } \\
\text { Chronic obstructive pulmonary } \\
\text { disease } \\
\text { Previous stroke } \\
\text { Diabetes mellitus } \\
\text { Cirrhosis }\end{array}$ & $\begin{array}{c}70(31.8 \%) \\
65(29.5 \%) \\
52(23.6 \%) \\
9(4 \%) \\
21(9.6 \%) \\
3(1.5 \%)\end{array}$ & $\begin{array}{c}30(39 \%) \\
14(18.2 \%) \\
20(25.9 \%) \\
3(3.9 \%) \\
9(11.7 \%) \\
1(1.3 \%)\end{array}$ & $\begin{array}{l}0.043 \\
0.192 \\
0.317 \\
1.000 \\
0.360 \\
1.000\end{array}$ \\
\hline $\begin{array}{l}\text { No. of comorbid conditions } \\
\qquad \begin{array}{c}0 \\
1 \\
2 \\
3+\end{array}\end{array}$ & $\begin{array}{c}47(26 \%) \\
65(35.9 \%) \\
56(31 \%) \\
13(7.1 \%)\end{array}$ & $\begin{array}{c}13(23,6 \%) \\
16(29 \%) \\
18(32.7 \%) \\
8(14.7 \%)\end{array}$ & 0.918 \\
\hline
\end{tabular}

Table 2: Pathological data observed in octogenarians' patients with gastric cancer.

\begin{tabular}{|c|c|c|c|}
\hline & Patients 80-85 Years & Patients > 85Years & P value \\
& $($ No.=181) & & \\
\hline Primitive tumo & & $6(10.9 \%)$ & 0.216 \\
cardias & $10(5.5 \%)$ & $34(61.9 \%)$ & 0.030 \\
pylorus & $80(44.3 \%)$ & $7(12.7 \%)$ & 0.001 \\
body & $64(35.3 \%)$ & $0(0 \%)$ & 0.122 \\
bottom & $10(5.5 \%)$ & $7(12.7 \%)$ & 0.014 \\
unspecified site & $6(3.4 \%)$ & $1(1.8 \%)$ & 0.304 \\
other & $11(6 \%)$ & $5(9 \%)$ & 0.489 \\
\hline Early & $25(14 \%)$ & $50(91 \%)$ & \\
\hline
\end{tabular}




\begin{tabular}{|c|c|c|c|}
\hline $\begin{array}{c}\text { Histological type } \\
\text { (Lauren's criteria) } \\
\text { Intestinal } \\
\text { Diffuse }\end{array}$ & $\begin{array}{c}103(57 \%) \\
78(43 \%)\end{array}$ & $\begin{array}{l}35(64 \%) \\
20(36 \%)\end{array}$ & 0.436 \\
\hline \multicolumn{4}{|c|}{ Lymph node metastasis (TNM) } \\
\hline Positive & $100(55 \%)$ & $33(60 \%)$ & 0.642 \\
\hline Negative & $81(45 \%)$ & $22(40 \%)$ & \\
\hline \multicolumn{4}{|l|}{ Stage TNM } \\
\hline I & $36(19.9 \%)$ & $4(7.4 \%)$ & \\
\hline II & $68(37.6 \%)$ & $27(49 \%)$ & 0.256 \\
\hline III & $51(28.2 \%)$ & $14(25.4 \%)$ & \\
\hline IV & $26(14.3 \%)$ & $10(18.2)$ & \\
\hline \multicolumn{4}{|l|}{ Grade } \\
\hline I & $15(8.3 \%)$ & $2(3.6 \%)$ & \\
\hline II & $43(23.8 \%)$ & $21(38.2 \%)$ & 0.116 \\
\hline III & $118(65.2 \%)$ & $32(58.2 \%)$ & \\
\hline IV & $5(2.7 \%)$ & $0(0 \%)$ & \\
\hline
\end{tabular}

Table 3: Surgical data of octogenarian's patients with gastric cancer.

\begin{tabular}{|c|c|c|c|}
\hline Parameters & $\begin{array}{l}\text { Patients 80-85 Years } \\
\qquad \text { (No.=181) }\end{array}$ & $\begin{array}{c}\text { Patients }>85 \text { Years } \\
\qquad(\text { No. }=55)\end{array}$ & $\mathbf{P}$ \\
\hline \multicolumn{4}{|l|}{ ASA score } \\
\hline II & $16(8.8 \%)$ & $2(3.6 \%)$ & \multirow{3}{*}{0.021} \\
\hline III & $140(77.4 \%)$ & $39(70.9 \%)$ & \\
\hline IV & $25(13.8 \%)$ & $14(25.5 \%)$ & \\
\hline \multicolumn{4}{|l|}{ Timing surgery } \\
\hline Elective & $118(65.2 \%)$ & $48(87.3 \%)$ & 0.001 \\
\hline Urgent & $63(34.8 \%)$ & $7(12.7 \%)$ & \\
\hline \multicolumn{4}{|l|}{ Surgery } \\
\hline Partial gastrectomy & $118(65.3 \%)$ & $38(69 \%)$ & 0.250 \\
\hline Total gastrectomy & $41(22.7 \%)$ & $7(12.7 \%)$ & 0.128 \\
\hline Gastroenterostomy alone & $11(6 \%)$ & $2(3.7 \%)$ & 0.738 \\
\hline Other & $11(8 \%)$ & $8(14.6 \%)$ & 0.052 \\
\hline \multicolumn{4}{|l|}{ Surgical approach } \\
\hline Roux & $59(32.6 \%)$ & $14(25.5 \%)$ & 0.405 \\
\hline Billroth II & $62(34.2 \%)$ & $14(25.5 \%)$ & 0.321 \\
\hline Other & $60(33.2 \%)$ & 27 (49\%) & 0.038 \\
\hline \multicolumn{4}{|l|}{ Lymph nodes dissection } \\
\hline D0 & $22(12.1 \%)$ & $10(18.1 \%)$ & 0.493 \\
\hline$<$ D2 & $15(8.2 \%)$ & $5(9 \%)$ & \\
\hline$>\mathrm{D} 2$ & $144(79.5 \%)$ & $40(72.7 \%)$ & \\
\hline
\end{tabular}




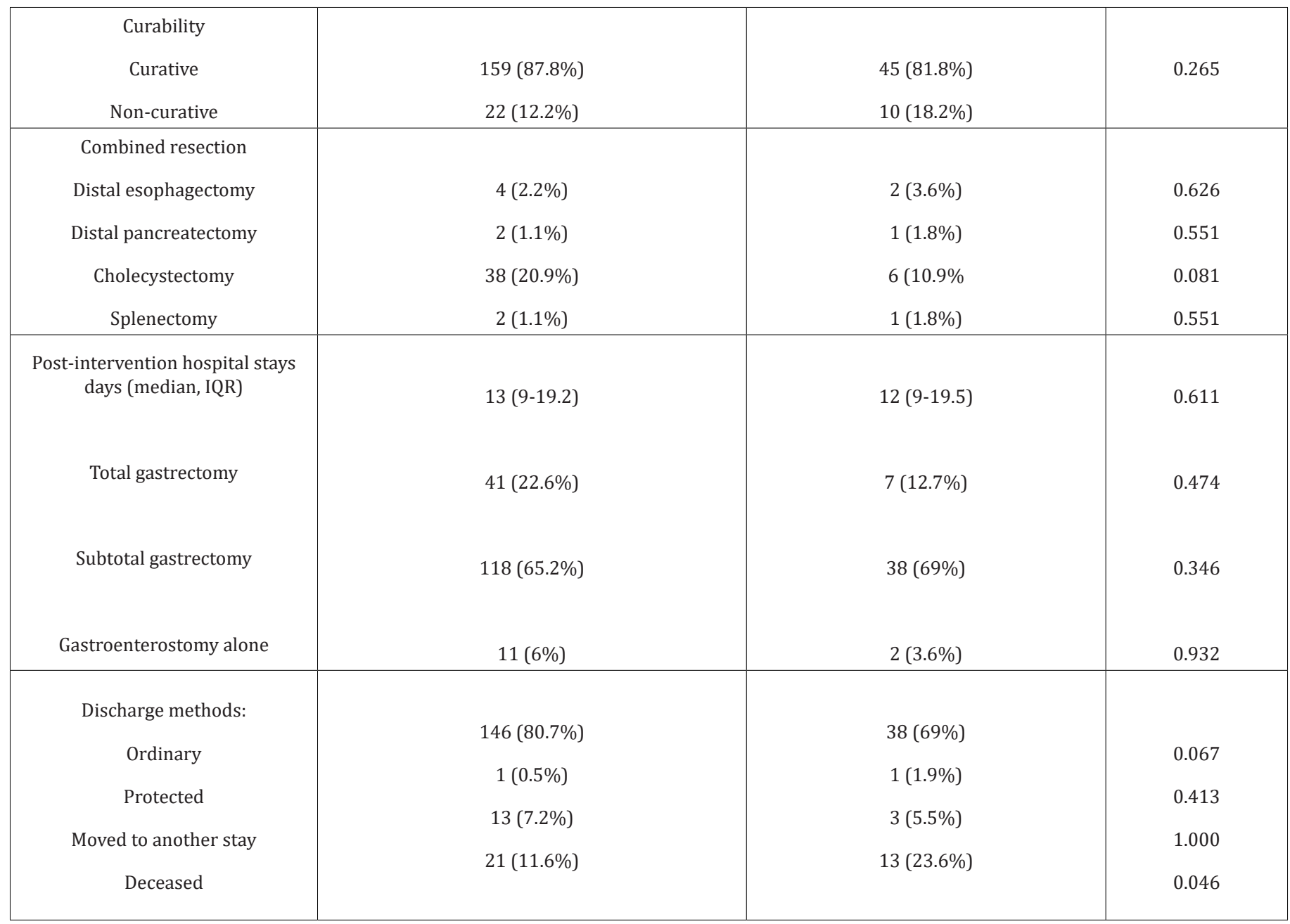

Table 4: Postoperative complications in octogenarian's patients after gastric surgery.

\begin{tabular}{|c|c|c|c|}
\hline & Patients 80-85 Years (No.=181) & $\begin{array}{c}\text { Patients }>85 \text { years } \\
(\text { No. }=55)\end{array}$ & $\mathbf{P}$ \\
\hline Overall postoperative & \multirow{2}{*}{$55(30.3 \%)$} & \multirow{2}{*}{$20(36.3 \%)$} & \multirow{2}{*}{0.413} \\
\hline complications & & & \\
\hline Medical & $40(72.7 \%)$ & $13(65 \%)$ & 0.854 \\
\hline Pleural effusion & 30 & 8 & \\
\hline Atelectasis & 7 & 3 & \\
\hline Pulmonary embolism & 1 & 1 & \\
\hline Stroke & 1 & 0 & \\
\hline Heart failure & 1 & 1 & \\
\hline Surgical & $15(28.3 \%)$ & $7(35 . \%)$ & 0.303 \\
\hline Anastomotic leakage & 2 & 1 & \\
\hline Intraabdominal abscess & 2 & 1 & \\
\hline Ileus & 2 & 0 & \\
\hline Cholecystitis & 1 & 0 & \\
\hline Bleeding & 1 & 1 & \\
\hline Wound infection & 3 & 1 & \\
\hline \multicolumn{4}{|c|}{ Clavien Score } \\
\hline$<3$ & $38(69 \%)$ & $9(45 \%)$ & 0.097 \\
\hline$>3$ & $17(31 \%)$ & $11(55 \%)$ & \\
\hline
\end{tabular}




\begin{tabular}{|l|c|c|c|}
\hline \multicolumn{4}{|c|}{ Mortality } \\
\hline at 30 days & $10(5.5 \%)$ & $5(9 \%)$ & 0.35 \\
\hline at 90 days & $11(6 \%)$ & $8(14.5 \%)$ & 0.052 \\
\hline
\end{tabular}

\section{Factors Associated to Post-Operative Mortality}

In the combined cohort, urgent surgery (OR 2.97 [95\% CI 1.47 - 6.02], p= 0.002) and ASA score >III (OR 3.12 [95\% CI 1.19 - 8.20], $\mathrm{p}=0.021)$ were statistically associated to post-operative mortality. The age ( OR 2.61 [95\% CI $0.91-7.49$ ], p = 0.074), the cardiac comorbidities (OR 0.53 [95\% CI 0.14-1.95], p=0.343) and the site of primary tumor (OR 1.07 [95\% CI 0.65-1.76], p = 0.775) were not identified as risk factors in the multi-variate logistic regression analysis.

\section{Survival}

Kaplan-Meier survival curves for combined cohort are displayed in Figures $1 \& 2$; the survival of patients with ASA score $>$ III and of patients submitted to urgent surgery was significantly worse than those of patients with ASA score $\leq$ III $(p=0.015)$ and of patients submitted to elective surgery ( $p=0.021$ ). Multivariable Cox regression analysis identified ASA score (HR 1.99, 95\% CI 1.02$3.86, \mathrm{p}=0.041$ ) and stage (HR 1.40, 95\% CI 1.07-1.82, $\mathrm{p}=0.012$ ) as independent prognostic factors that significantly influenced survival.

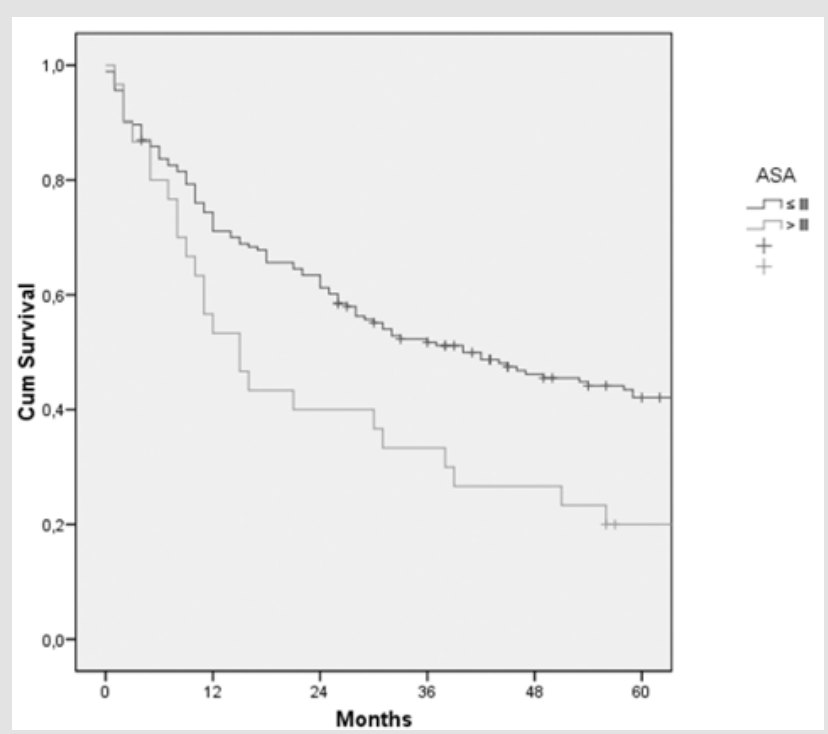

Figure 1: Kaplan-Meier survival curve in patients with ASA score $>$ III and $\leq$ III. Log- rank test $\mathrm{p}=0.015$.

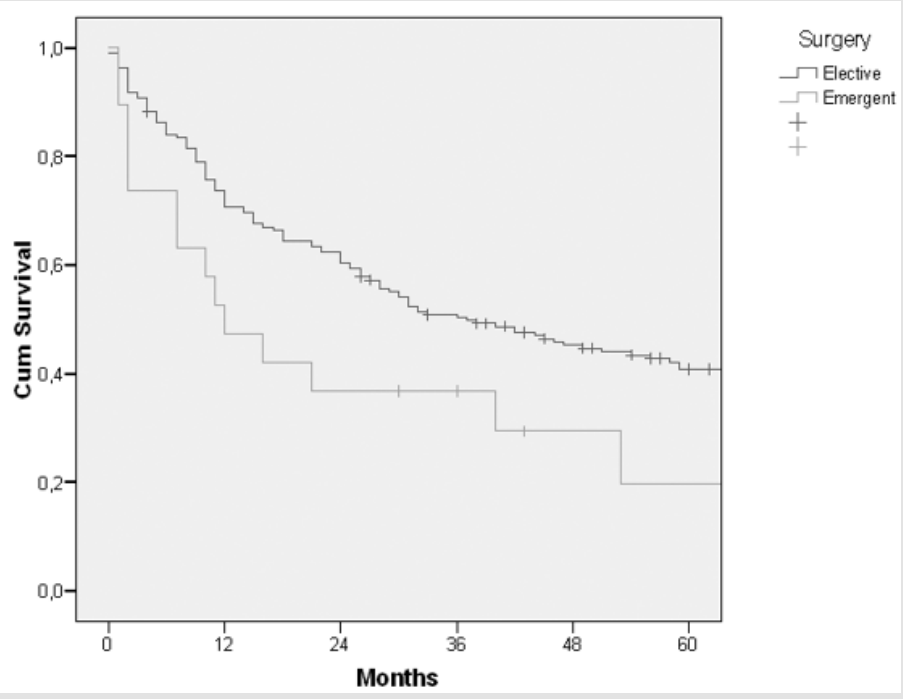

Figure 2: Kaplan-Meier survival curve in patients submitted to elective and urgent surgery. Log- rank test $p=0.021$. 


\section{Discussion}

Gastric cancer is thought to occur more frequently in men. The high number of women $(60 \%)$ in this study is probably due to the lower life expectancy of men. The current study demonstrates that clinic-pathological features of gastric cancer such as histological type, tumor site, disease extent and pathological stage were neither related to patient age nor to surgical outcome. The majority of the patients $(87 \%)$ had the tumor at a stage greater than I. It is plausible that the observation is related to the lower attention, for the elderly, to preventive health programs for the detection of cancer at an early stage com-pared to what occurs in high-risk countries. In the present study, $87.3 \%$ of the $>85$ years old underwent elective surgery. It appears likely that in most cases the clinical presentation of the disease allowed planning an elective procedure. On the other hand, $34.8 \%$ of the $80-85$ years old were operated following an urgent surgical presentation. The possible explanation is that, in these patients, the diagnosis was frequently triggered by a complication manifestation. Among the $>85$ years old of our report, $25.5 \%$ of the cases had an ASA score>III. ASA scores differed significantly be-tween two group patients. Most likely, either the $>85$ years old arrived at the pre-operative visit with a worse functional deficit and due to more careful preoperative assessments concerning organ function and performance status.

In our report, the rate of postoperative mortality in $>85$ years old was significantly higher than in 80-85 years old (9\% versus $5.5 \%$ ). Multivariate analysis of the combined cohort indicated however that the age>85 did not correlate with mortality; on the contrary, urgent surgical presentation and an ASA score>III were associated with postoperative mor-tality and with one-year surgery survival. In the combined cohort, ASA>III and urgent surgery were associated with postoperative mortality. In literature, there is wide consensus on the causal relation "ASA - post-operative mortality" for elderly patients who have undergone gastric cancer surgery $[12,13]$. According to our experience, in the oldest old, the higher ASA score may correlate to lower functional reserves and more significant comorbidities, enough to influence short-term outcomes. The correlation between urgent surgical presentation and operative mortality, for the oldest old undergoing gastric can-cer surgery, is confirmed in literature [14]. The hypothesis is that bowel obstruction or perforation may carry a poor prognosis independently from the surgical operation; it is possible that the surgical procedure physical and mental stress along with the general anaesthesia further influence short-term outcomes in such frail conditions. According to several observations, important predictors of postoperative mortality in oldest old patients who have un-dergone surgery for gastric cancer are ASA score and urgent surgical presentation [15]. Age did not represent a factor associated with postoperative mortality in previous reports [16-18].

Considering these results, it appears appropriate planning prospective studies in order to achieve even stronger evidence regarding the safety of the oldest old undergo-ing gastric cancer surgery. Age did not represent an independent risk factor for postoperative mortality; patients should be assessed according to their general physical condition, whatever the age. In several studies similar to our own, ASA score and albumin serum concentration are reported as indirect indicators of the patient's functional conditions [19-21]. In recent research, multidimensional geriatric assessment has shown to be useful in giving a $360^{\circ}$ picture of a patient's status, in terms of physical and functional condition [22,23]. Thus, the combination of geriatric assessment and consolidated prognostic indicators, such as the TNM staging, could lead to more effective algorithms for preoperative risk stratification. Moreover, further studies should focus on confirming the clinical usefulness of functional variables (e.g. ASA score) as postoperative mortality predictors. Keeping in mind what mentioned above, a clinical intervention, aiming to modify the above mentioned biological and functional aspects, might improve short-term outcomes. However, since most of the surgeries on nonagenarians are performed in an emer-gent status, significant results for nutritional interventions might be difficult to achieve before surgery.

This study has the limitations of retrospective investigations involving chart review. Moreover, given the rarity of an operation on a nonagenarian, the number of the sample might limit the statistical power of and limit the generalization of the conclusions. A further limitation of our report was the lack of a standardized institutional protocol for postopera-tive care.

\section{Conclusion}

From the current analysis of a large database of 236 over octogenarians' patients who have undergone gastric cancer surgery, urgent surgical presentation and ASA score $>$ III, but not age $\geq 85$, were correlated to postoperative mortality. These results should prompt an improvement in multidimensional geriatric assessment, to better define the risk stratifi-cation in the oldest old patients undergoing gastric surgery. Moreover, the development of effective corrective interven-tions of the above preoperative functional variables might lead to an improvement of surgical outcomes in the increas-ingly large geriatric population.

\section{Compliance with Ethical Guidelines}

Conflict of interest Lugaresi M, Duchi A, Di Saverio S, Argento F, Yavuzdiler KE, Martini F, Novello M, Cavallari G, Nar-do B declare that they have no competing interests.

\section{References}

1. Ferlay J, Soerjomataram I, Ervik M, Dikshit R, Eser S, et al. (2013) GLOBOCAN 2012 v1.0, Cancer Incidence and Mortality Worldwide: IARC CancerBase No. 11. Lyon, France: International Agency for Research on Cancer.

2. (2018) Eurocare survival of cancer patients in Italy.

3. Parkin DM, Pisani P, Ferlay J (1999) Estimates of the worldwide incidence of 25 major cancers in 1990. Int J Cancer 80(6): 827-841. 
4. (2011) WHO. Global health and aging. World Health Organization.

5. (2018) Istituto Nazionale di Statistica.

6. Edge SB, April Fritz G, David Byrd R, Frederick Greene L (2009) American Joint Committee on Cancer, AJCC cancer staging manual $\left(7^{\text {th }}\right.$ Edn.)., Springer Verlag, New York, USA.

7. Fleshman J, American Society of Colon and Rectal Surgeons (2007) The ASCRS Textbook of Colon and Rectal Surgery. Springer, New York, NY, USA.

8. (2014) ASA Physical Status Classification System. American Society of Anesthesiologists.

9. Dindo D, Demartines N, Clavien PA (2004) Classification of Surgical Complications: A New Proposal With Evaluation in a Cohort of 6336 Patients and Results of a Survey. Annals of Surgery 240(2): 205-213.

10. (1988) Japanese Gastric Cancer Association. Japanese classification of gastric carcinoma (13 ${ }^{\text {th }}$ Edn.)., Kanehara \& Co., Ltd, Tokyo, Japan.

11. (2010) Japanese Gastric Cancer Association. Japanese classification of gastric carcinoma (14 ${ }^{\text {th }}$ Edn.)., Kanehara, Tokyo, Japan.

12. Takeuchi D, Koide N, Suzuki A, Ishizone S, Shimizu F, et al. (2015) Postoperative complications in elderly patients with gastric cancer. J Surg Res 198(2): 317-326.

13. Takama T, Okano K, Kondo A, Akamoto S, Fujiwara M, et al. (2015) Predictors of postoperative complications in elderly and oldest old patients with gastric cancer. Gastric Cancer 18(3): 653-661s.

14. Fecho K, Lunney AT, Boysen PG, Rock P, Norfleet EA (2008) Postoperative mortality after inpatient surgery: Incidence and risk factors. Ther Clin Risk Manag 4(4): 681-688.

15. Van Stijn MF, Korkic Halilovic I, Bakker MS, van der Ploeg T, Van Leeuwen PA, et al. (2013) Preoperative nutrition status and postoperative

ISSN: 2574-1241

DOI: $10.26717 /$ BJSTR.2020.24.004106

Bruno Nardo. Biomed J Sci \& Tech Res

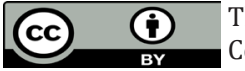

This work is licensed under Creative Commons Attribution 4.0 License

Submission Link: https://biomedres.us/submit-manuscript.php outcome in elderly general surgery patients: a systematic review. JPEN J Parenter Enteral Nutr 37(1): 37-43.

16. Samet J, Hunt WC, Key C, Humble CG, Goodwin JS (1986) Choice of cancer therapy varies with age of patient. JAMA 255: 3385-3390.

17. Bausys Rimantas, Augustinas Bausys, Indre Vysniauskaite, Kazimieras Maneikis, Eugenijus Stratilatovas, et al. (2018) Surgical Treatment Outcomes of Patients with T1-T2 Gastric Cancer: Does the Age Matter When Excellent Treatment Results Are Expected? World J Surg Oncol 16(1): 79.

18. Pisanu, Montisci, Piu, Uccheddu (2007) Curative surgery for gastric cancer in the elderly: treatment decisions, surgical morbidity, mortality, prognosis and quality of life. Tumori 93(5): 478-484.

19. Kim MS, Kim S (2016) Outcome of Gastric Cancer Surgery in Elderly Patients. J Gastric Cancer 16(4): 254-259.

20. Nelen SD, Bosscha K, Lemmens VEPP, Hartgrink HH, Verhoeven RHA, et al. (2018) Morbidity and mortality according to age following gastrectomy for gastric cancer. Br J Surg.

21. Gupta D, Lis CG (2010) Pretreatment serum albumin as a predictor of cancer survival: a systematic review of the epidemiological literature. Nutr J 9: 69 .

22. Extermann M, Aapro M, Bernabei R, Cohen HJ, Droz JP, et al. (2005) Use of comprehensive geriatric assessment in older cancer patients: recommendations from the task force on CGA of the International Society of Geriatric Oncology (SIOG). Crit Rev Oncol Hematol 55(3): 241-252.

23. Wildiers H, Heeren P, Puts M, Topinkova E, Janssen Heijnen ML, et al. (2014) International Society of Geriatric Oncology consensus on geriatric assessment in older patients with cancer. J Clin Oncol 32(24): 2595-2603.

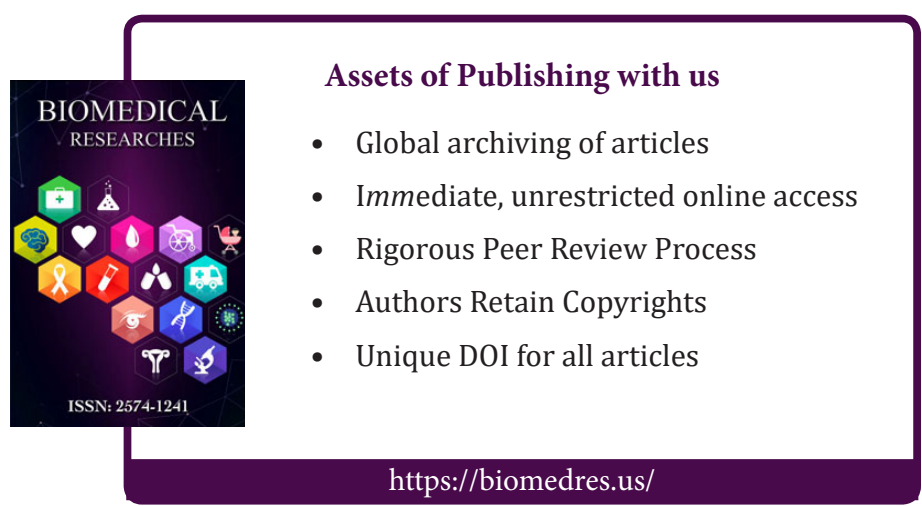

